\section{The utility of visual function questionnaire in the assessment of the impact of diabetic retinopathy on vision-related quality of life}

A Gabrielian'1, SM Hariprasad', RD Jager',

JL Green ${ }^{1}$ and WF Mieler ${ }^{2}$ emotional aspects of the disease as well as visual function. Subjects with PDR vs NPDR suffer significant loss of VRQOL.

Eye (2010) 24, 29-35; doi:10.1038/eye.2009.56;

published online 27 March 2009

Keywords: quality of life; diabetic retinopathy; Visual Function Questionnaire-25 (VFQ-25); utility; Vision Preference Value Scale (VPVS)

\section{Introduction}

Diabetic retinopathy is the leading cause of blindness among the working age population (20-74 years) in the United States. ${ }^{1}$ Every year, approximately 12000-24000 diabetic individuals develop visual loss, making diabetes responsible for $12 \%$ of new cases of legal blindness each year. ${ }^{2}$

Patients with diabetic retinopathy suffer a significant decrease in quality of life as retinopathy can seriously impair visual function. ${ }^{3,4}$ Traditionally, ophthalmologists have relied on Snellen visual acuity (VA) to gauge visual function and vision-related quality of life. Although VA is an objective measure of macular function, in recent years, it has been shown that the eye diseases can affect various aspects of vision beyond acuity, such as glare, contrast sensitivity, colour vision, and stereoscopic vision. ${ }^{5,6}$

Recognizing that VA alone fails to capture the impact of vision disability, the National Eye Institute Visual Function Questionnaire-25 (VFQ) was developed to measure self-reported,
${ }^{1}$ Department of Surgery, Section of Ophthalmology and Visual Sciences, University of Chicago, Chicago, IL, USA

${ }^{2}$ Department of Ophthalmology and Visual Sciences, University of Illinois Eye and Ear Infirmary, Chicago, IL, USA

Correspondence: SM Hariprasad, Associate Professor and Director of Clinical Research, Department of Surgery, Section of Ophthalmology and Visual Science, University of Chicago, 5841 South Maryland, MC 2114

Chicago,

IL 60637 . USA

Tel: + 773702 6110; Fax: + 7738343782 E-mail: retina@ uchicago.edu

Received: 30 December 2008

Accepted in revised form: 19 February 2009 Published online: 27 March 2009

Conflict of interest: The authors declare no conflict of interest. 
vision-related aspects of health status that are most significant to individuals with chronic eye disease. ${ }^{7-9}$ To this end, the VFQ survey assesses the ability to perform vision-related daily activities and the impact of visual impairment on emotional and social domains.

Although in theory VFQ scores measure vision-related quality of life (VRQOL) more accurately than VA, to the best of our knowledge, no report to date has yet proven this to hold true in practice. The purpose of the study was to assess whether the VFQ is indeed a more accurate measure of VRQOL than VA in patients with diabetic retinopathy. We hypothesize that the superiority of VFQ will lie in its ability to capture a fuller representation of diabetic retinopathy's impact on VRQOL due to the mental, emotional, and social impact of the disease. We predict that this will be especially apparent as we use the VFQ to compare the loss of VRQOL in patients with PDR vs NPDR.

\section{Materials and methods}

The study population consisted of 104 subjects with diabetic retinopathy examined at the University of Chicago's, Section of Ophthalmology and Visual Science. Potential subjects were identified through diagnosis codes of billing records indicating the stage of diabetic retinopathy. Exclusion criteria included current presence of clinically significant and active diabetic macular oedema, and presence of any ocular comorbidity.

\section{Data collection}

The two questionnaires directed to measure VRQOL - VFQ and Vision Preference Value Scale (VPVS) - were administered in a standardized manner in the aforementioned order by telephone. Following the administration of the surveys, the interviewer ascertained patient demographics and history of diabetes information that could potentially act as covariates to VRQOL. Demographic information noted included ethnicity, sex, age, education, and insurance coverage. History of diabetes information noted included insulin use and duration of diabetes.

Medical records review detailed potential ophthalmic covariates to VRQOL, such as additional ocular conditions, vision-related treatments, and other health related comorbidities. Ocular conditions noted included strabismus, glaucoma, cataracts, and retinal detachment. Vision-related treatments noted included pars plana vitrectomy, epiretinal membrane peel, focal laser, panretinal photocoagulation (PRP) laser, and intraocular injections. Other comorbidities noted included hypertension, hyperlipidaemia, amputation, renal failure, arthritis, cardiovascular and pulmonary conditions, and anxiety and depression. Clinical measures recorded included $\mathrm{HbA} 1 \mathrm{C}$ levels and bestcorrected VAs (BCVAs) from the medical record.

\section{Definitions and scoring}

The severity of retinopathy was noted from diagnoses codes used for billing. Subjects classified as type 1 diabetics were diagnosed with diabetes at younger than 30 years of age and remained insulin-dependent. Subjects with $\mathrm{HbA} 1 \mathrm{C}$ levels above $8 \%$ were classified as high-risk for visual impairment.

BCVAs, as measured by Snellen VA charts, were extracted from medical records and were converted to express the logarithm of the minimum angle of resolution (LogMAR). In addition, a weighted LogMAR incorporating the vision of both eyes was calculated. Following the suggestion of the AMA's Guides to the Evaluation of Permenant Impairment, the weighted average gives a factor of 0.75 to the better eye and a factor of 0.25 to the worse eye. ${ }^{5}$ In calculating LogMAR scores, counting fingers were assigned a Snellen VA of 20/1000, hand motion 20/2000, and light perception or no light perception $20 / 4000 .^{10}$

The VFQ's reliability and validity has been tested and confirmed on a heterogeneous group of patients with eye conditions, such as age-related macular degeneration, glaucoma, cataract, viral retinitis, and diabetic retinopathy. ${ }^{8}$ The VFQ consists of 25 vision-targeted questions plus 1 overall health rating question. The questions fall into 11 vision-specific subscales: general vision, ocular pain, near activities, distance activities, social functioning, mental health, role difficulties, dependency, driving, colour vision, and peripheral vision. The VFQ takes $10 \mathrm{~min}$ to complete.

The VPVS is a rating scale and represents one of many ways to measure utility values. In contrast to the VFQ, which measures a person's perception of his/her visual function, and hence is a more direct reflection of the person's disease state, the VPVS measures one's preference for function, and therefore, is a more abstract measure of visual function. The VPVS rating scale is easy to understand and is able to produce valid and reliable results without visual aids or an in-person interview. ${ }^{11}$ In addition, the VPVS has had good test-retest reliability and internal consistency in most studies. ${ }^{12,13}$ As a result, the VPVS was used as the gold standard for the assessment of visual function in this experiment. Other methods of assessing utility include the standard gamble, time-trade off, and willingness to pay methods. These other methods of measuring utility values may be more susceptible to the influence of the subjects' socioeconomic or educational background. ${ }^{14,15}$ 
The VPVS requires subjects to rate their current vision on a scale of $0-100$, where 0 represents blindness and 100 represents perfect vision. Subjects then rate how they would feel about their current health state if they were completely blind on a scale of $0-100$, where 0 represents death and 100 represents perfect health with perfect vision. Finally, they rate how they would feel about their current health if they had perfect vision on the same scale. These three answers yield a utility score on a scale from 0 to 1, measuring the patient's overall VRQOL. ${ }^{11}$ The VPVS, like the VFQ, takes about 10 min to complete.

The general health status was assessed through the single item general ratings question on the VFQ. This question has shown a strong predictive power for future health and mortality in population-based studies. ${ }^{8}$

\section{Statistical analysis}

Descriptive statistics were used to classify subjects according to key demographic and clinical data. All statistical analyses were carried out using SPSS software.

To determine whether VFQ is a more accurate measure of VRQOL than VA while using VPVS scores as the standard, we first considered a univariate analysis. Pearson's correlation coefficients measured the strength of relationships between the BCVA of the better eye, the weighted BCVA (WVA), and the VFQ scores with VPVS values. Univariate linear regression was used to confirm the results of Pearson's correlation.

Next, we turned to multivariate linear models to see whether the same conclusions could be drawn when controlling for potential covariates to VRQOL. However, as there were a large number of potential explanatory variables, we began with data mining, using stepwise linear regression to determine which pieces of demographic and clinical information were covariates to the VFQ and VPVS scores. We set the entry criteria as $P<0.1$ and the removal criteria as $P>0.2$. The potential covariates screened included the following: age, sex, insurance status (medicaid, medicare, and private), ethnicity (Caucasian, African American, Hispanic, and Asian), education level, general health status, general health comorbidities (hypertension, hyperlipidaemia, cardiovascular, pulmonary, and renal, arthritis), ocular comorbidities (cataract and epiretinal membrane), ocular treatment (cataract extraction, pars plana vitrectomy, epiretinal membrane peel, panretinal photocoagulation laser, and focal laser), haemoglobin A1C (HbA1C), duration of diabetes, insulin use, and type of diabetes. As standard variables to control in a VRQOL outcomes study, we included age, sex, total ocular comorbidities, and general health status, in addition to variables pulled from the stepwise linear regression (duration of diabetes, private insurance, hypertension, insulin use, PRP treatment, and renal comorbidity). All these covariates together were denoted as 'base' covariates.

After determining the covariates to VRQOL, we created four multivariate linear regression models in which VPVS was always assigned as the dependent factor. In the first model, the base covariates were assigned as the only independent factors. In the second, third and fourth models, the base covariates plus either the BCVA, WVA, or VFQ scores, respectively, were assigned the independent factors. Comparison of the four models allowed us to determine which factor best explained the variance observed in VPVS.

To determine why VFQ was a more accurate VRQOL measure for patients with diabetic retinopathy, we measured the absolute Spearman correlation coefficients between VPVS values with each of the VFQ subscale scores to determine which VFQ subscales correlated strongest with patient's health state preference due to diabetic retinopathy.

Finally, analysis was carried out to compare the VRQOL of patients with NPDR vs PDR using VFQ as our measure. We created 12 multivariate linear regression models in which base covariates and the stage of retinopathy (PDR or NPDR) were always assigned as the independent factors. Each model was assigned either a VFQ subscale or the total VFQ score as the dependent factor.

\section{Results}

Of 125 consecutive patients invited to participate in the study, 104 consented to participate. Patients who refused to participate tended to be older than patients who consented. Those who refused had a mean age of 68.3 (SD: 9.1), whereas those who participated had a mean age of 59.7 years (SD: 13.2). The study population was predominantly African American, women, and diagnosed with type 2 diabetes. Overall, the population was evenly divided among the subjects diagnosed with NPDR vs PDR according to the ETDRS classification (Table 1).

In Table 2, the absolute Pearson's correlation coefficient values show a markedly stronger correlation coefficient between VPVS and VFQ than between VPVS and BCVA or WVA. This shows that VFQ appears to be a more accurate measure of VRQOL than VA.

A univariate linear regression reiterates these findings when VPVS is assigned as the dependent variable and either VFQ, BCVA, or WVA are assigned as the predictor: BCVA $\left(R^{2}=0.07\right)$, WVA $\left(R^{2}=0.11\right)$, and VFQ $\left(R^{2}=0.24\right)$.

The exploratory stepwise linear regression to screen for potential covariates of VRQOL determined that duration of diabetes, private insurance, hypertension, and general health were dependent factors of the VPVS 
Table 1 Distribution of subjects by demographics, ocular pathology, treatment factors, and medical factors

\begin{tabular}{|c|c|}
\hline Factor & $\begin{array}{c}\text { Number of al } \\
\text { subjects (\%) }\end{array}$ \\
\hline \multicolumn{2}{|l|}{ Demographics } \\
\hline Female & $74(71)$ \\
\hline Male & $30(29)$ \\
\hline African American & $86(83)$ \\
\hline Caucasian, Hispanic, Asian & $18(17)$ \\
\hline \multicolumn{2}{|l|}{ Age at VFQ (years) } \\
\hline$<40$ & $8(8)$ \\
\hline $40-50$ & $15(14)$ \\
\hline $51-60$ & $29(28)$ \\
\hline $61-70$ & $29(28)$ \\
\hline$>70$ & $23(22)$ \\
\hline \multicolumn{2}{|l|}{ Insurance } \\
\hline Medicaid & $38(37)$ \\
\hline Medicare & $59(48)$ \\
\hline Private & $58(56)$ \\
\hline \multicolumn{2}{|l|}{ Education } \\
\hline High school/GED & $43(41)$ \\
\hline Some college & $39(38)$ \\
\hline College degree & $9(9)$ \\
\hline Graduate school & $8(8)$ \\
\hline \multicolumn{2}{|l|}{ Diabetes } \\
\hline Type 1 diabetes & $15(14)$ \\
\hline Type 2 Diabetes & $89(86)$ \\
\hline \multicolumn{2}{|l|}{ Duration (years) } \\
\hline$<10$ & $19(23)$ \\
\hline $10-14$ & $14(17)$ \\
\hline $15-19$ & $14(17)$ \\
\hline$>20$ & $34(41)$ \\
\hline Insulin use & $63(60)$ \\
\hline $\mathrm{HbA} 1 \mathrm{c}>8 \%$ & $37(36)$ \\
\hline \multicolumn{2}{|l|}{ Ocular pathology } \\
\hline \multicolumn{2}{|l|}{ Diabetic retinopathy } \\
\hline Mild NPDR & $27(26)$ \\
\hline Moderate NPDR & $20(19)$ \\
\hline Severe NPDR & $4(4)$ \\
\hline Total NPDR & $51(49)$ \\
\hline PDR & $53(51)$ \\
\hline \multicolumn{2}{|l|}{ Ocular comorbidity } \\
\hline Cataract & $41(40)$ \\
\hline Epiretinal membrane & $5(5)$ \\
\hline \multicolumn{2}{|l|}{ Prior ocular treatment } \\
\hline PRP laser & $53(51)$ \\
\hline Focal laser & $4(4)$ \\
\hline Pars plana vitrectomy & $25(24)$ \\
\hline Pars plana vitrectomy with membrane peeling & $10(10)$ \\
\hline Cataract extraction & $11(11)$ \\
\hline \multicolumn{2}{|l|}{ General health comorbidity } \\
\hline Hypertension & $80(77)$ \\
\hline Hyperlipidaemia & $28(27)$ \\
\hline Cardiac & $17(16)$ \\
\hline Pulmonary & $20(19)$ \\
\hline Renal & $12(12)$ \\
\hline Arthritis & $25(24)$ \\
\hline
\end{tabular}

$\mathrm{VFQ}=$ visual function questionnaire; $\mathrm{NPDR}=$ non-proliferative diabetic retinopathy; $\mathrm{PDR}=$ proliferative diabetic retinopathy; $\mathrm{PRP}=$ panretinal photocoagulation.
Table 2 Pearson's correlation between VRQOL constructs

\begin{tabular}{ll}
\hline & $V P V S$ \\
\hline BCVA & $0.264^{\mathrm{a}}$ \\
WVA & $0.333^{\mathrm{a}}$ \\
VFQ & $0.494^{\mathrm{a}}$
\end{tabular}

$\mathrm{BCVA}=$ best-corrected visual acuity; $\mathrm{VFQ}=$ visual function questionnaire; $\mathrm{VRQOL}=$ vision-related quality of life; $\mathrm{WVA}=$ weighted $\mathrm{BCVA}$.

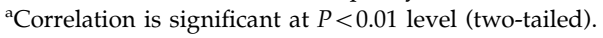

Table 3 Multivariate linear regression of VRQOL, with VPVS as the dependent factor

\begin{tabular}{llc}
\hline Model & Independent factors & $\mathrm{R}^{2}$ \\
\hline 1 & Base & 0.186 \\
2 & Base + BCVA & 0.213 \\
3 & Base + WVA & 0.238 \\
4 & Base + VFQ & 0.324 \\
5 & Base + WVA + VFQ & 0.325 \\
\hline
\end{tabular}

$\mathrm{BCVA}=$ best-corrected visual acuity; $\mathrm{VFQ}=$ visual function questionnaire; $\mathrm{VPVS}=$ vision preference value scale; $\mathrm{VRQOL}=$ vision-related quality of life; WVA = weighted BCVA.

score $\left(P<0.1, R^{2}=0.251\right)$. It was also determined that insulin use, prior PRP laser treatment, renal comorbidity, and general health were dependent factors of the VFQ score $\left(P<0.1, R^{2}=0.386\right)$.

Our final linear regression model examining the relationship of various VRQOL instruments included the following 10 covariates as independent factors: standard covariates in VRQOL outcomes studies (age, sex, general health, and total ocular comorbidity), covariates of VPVS (duration of diabetes, private insurance, hypertension, and general health), and covariates of VFQ (insulin use, PRP treatment, renal comorbidity, and general health). These 10 variables were referred to collectively as the base covariates as mentioned above.

The multivariate linear regression corroborated our findings from the univariate analysis (Table 3). WVA explained more of the variance of VPVS than BCVA. However, the model assigning VFQ as an independent factor (model no. 4) explained the greatest proportion of variance in the VPVS scores.

Spearman correlation of VFQ subscales with VPVS scores explained why VFQ is a better measure of VRQOL than VA (Table 4). The largest absolute correlation coefficients and the strongest correlations between VPVS and VFQ subscales were in the following categories: role difficulties, near activities, distance activities, mental and dependency subscales.

With the VFQ as the more accurate instrument, we evaluated the difference in VRQOL in subjects with NPDR vs PDR; substantial differences between these two groups were found (Table 5). 
Table 4 Spearman correlation between VPVS and VFQ subscales

\begin{tabular}{lc}
\hline VFQ subscales & $V P V S$ \\
\hline Ocular pain & 0.33 \\
Near activities & 0.44 \\
Distance activities & 0.43 \\
Social & 0.34 \\
Mental & 0.43 \\
Role differences & 0.50 \\
Dependence & 0.38 \\
Driving & $0.17^{\mathrm{a}}$ \\
Color & 0.30 \\
Peripheral vision & 0.37 \\
\hline
\end{tabular}

$\mathrm{VFQ}=$ visual function questionnaire; $\mathrm{VPVS}=$ vision preference value scale.

Correlation is significant at the 0.01 level (two-tailed) for all unmarked results.

${ }^{\mathrm{a} C}$ Correlation is not significant at the 0.05 level.

Table 5 Point differences in VFQ total and subscales for NPDR vs PDR subjects, with base + stage of retinopathy as independent factors for all models

\begin{tabular}{clccc}
\hline Model & Dependent factor & $\mathrm{R}^{2}$ & $\begin{array}{c}\beta \text { Coefficient }^{a} \\
(95 \% \text { CI })\end{array}$ & P-value \\
\hline 1 & Total VFQ & 0.48 & $26.1(15.6-36.7)$ & $<0.01$ \\
2 & General vision & 0.38 & $18(6.4-29.6)$ & $<0.01$ \\
3 & Ocular pain & 0.20 & $9.8(1.5-18.1)$ & $<0.05$ \\
4 & Near activities & 0.43 & $32(19.4-44.5)$ & $<0.01$ \\
5 & Distance Activities & 0.35 & $24.7(10.4-39.1)$ & $<0.01$ \\
6 & Social & 0.31 & $27.3(12.9-41.5)$ & $<0.01$ \\
7 & Mental & 0.50 & $34.2(18.9-45.5)$ & $<0.01$ \\
8 & Role differences & 0.39 & $30.2(13.7-46.7)$ & $<0.01$ \\
9 & Dependence & 0.40 & $30.7(12.7-48.6)$ & $<0.01$ \\
10 & Driving & 0.19 & $8.6(-19.2-36.5)$ & $\mathrm{NS}$ \\
11 & Colour & 0.27 & $19.8(8.2-31.3)$ & $<0.01$ \\
12 & Peripheral & 0.35 & $33.6(18.0-49.2)$ & $<0.01$ \\
\hline
\end{tabular}

$\mathrm{VFQ}=$ visual function questionnaire; $\mathrm{NPDR}=$ non-proliferative diabetic retinopathy; $\mathrm{NS}=$ not significant; $\mathrm{PDR}=$ proliferative diabetic retinopathy ${ }^{\mathrm{a}} \beta$ Coefficient is for the stage of retinopathy (NPDR $v s$ PDR) and reflects the point difference in VFQ (100-point scale).

Among some of the VFQ subscales with the strongest correlation to VPVS (role differences, dependence, and mental), subjects with PDR scored approximately 30 points less than their NPDR counterparts. The visual function subscales (near and distance activities) revealed a mean reduction of 25-30 points in subjects with PDR us NPDR.

\section{Discussion}

As we aim for patient-centered and cost-effective healthcare, VRQOL is increasingly becoming pertinent to shaping the standard of care. In recent years, there has been a move in the retina community to use the VFQ over VA in assessing VRQOL. However, to the best of our knowledge, to date, there is no report showing VFQ to be a superior measure. ${ }^{16}$ Understanding and measuring VRQOL in an accurate manner is particularly significant as clinicians consistently underestimate the impact of ocular disease on their patients' lives. ${ }^{17}$

We chose the VPVS as the gold standard for several reasons. The VPVS allows the patients to fully express their perception of how much impact they feel their visual impairment has had on their lives. The final VPVS score expresses the subject's overall sense of well-being, energy or enjoyment in life, as impacted by diabetic retinopathy. The VPVS also serves as a solid standard because it yields utility scores, which are the basis for incorporating quality of life measures into cost-benefitrisk analyses. ${ }^{18,19}$ Finally, VPVS historically has been shown to have good test-retest reliability and internal consistency in numerous studies due to its unique rating scale technique. ${ }^{12,13}$ The rating scale technique can be expected to yield reasonable response rates because it is relatively easy for most people to understand. It yields reliable and valid results, even without an in-person interview or visual aids. ${ }^{13}$ At a time when 1 out of every 10 healthcare dollars in the United States is spent on diabetes and its complications, cost-effective diabetic healthcare is a pertinent issue. ${ }^{20}$

This is the first report showing VFQ as a superior measure of VRQOL in patients with diabetic retinopathy. We showed that VA does not reflect the extent to which vision-related function is impaired due to diabetic retinopathy. Patients with PDR may experience a wide range of symptoms from floaters to distortions and blurriness, to temporary or permanent loss of central or peripheral vision. These visual symptoms may not be fully addressed by VA, which is more reflective of macular function than of overall visual function. ${ }^{21}$

Furthermore, one of the VFQ's greatest strengths over VA is in its assessment of the degree of anxiety, fear, and mental anguish associated with diabetic retinopathy. Interestingly, the subscale with the biggest decrease as retinopathy progresses from NPDR to PDR was visionspecific mental health. The mental health subscale captures the worry, frustration, lack of control over activities, and the fear of potential embarrassment associated with eyesight. This finding reiterates the impact of diabetic retinopathy on emotional well-being and security in addition to visual function.

Potential limitations to our study include the systematic bias introduced by non-consenting patients. In general, patients who refused to participate were older, but there may be other trends among this subset that could have skewed our results. Another limitation to our study is that we did not factor in the individual 
subjects' rate of progression of visual loss, the extent of disparity in visual functioning between the two eyes, or the patients' use of low vision services. Demographically, our patient population was mostly African American and women, which is not necessarily representative of the general population. All of these factors may have influenced our subjects' attitudes towards visual disability. We also did not factor in the focusing effect of the order of administration of the VFQ and VPVS questionnaires. ${ }^{22}$

Focus groups have reported patient concerns over loss of independence, mobility, leisure and self-care activities, but have failed to quantify this impact in a manner amenable to guiding healthcare decisions. ${ }^{23}$ The VFQ's superiority lies in its ability to accurately measure this adverse impact. The dramatic decline in VRQOL observed in patients with PDR vs NPDR, coupled with the high prevalence of diabetes, suggests that treatments aiming to slow the progression of diabetic retinopathy merit attention. The VFQ is an instrument that can provide clinicians and policymakers with a more accurate assessment of the worth of specific healthcare interventions, and can be a useful adjunct to the regular eye examination.

\section{Acknowledgements}

This study was supported by funding from the University of Chicago Pritzker School of Medicine, Chicago, IL, USA. This study was carried out with the approval from the University of Chicago School of Medicine Institutional Review Board (IRB), and therefore has been performed in accordance with the ethical standards laid down in the 1964 Declaration of Helsinki. Oral informed consent was obtained from all subjects before enrollment in the study, and the study is in accordance with HIPAA regulations. This study was completed with statistical consultation from Yang Sheny (University of Chicago).

\section{References}

1 Congdon N, O'Colmain B, Klaver CC, Klein R, Muñoz B, Friedman DS et al. Eye Diseases Prevalence Research Group. Causes and prevalence of visual impairment among adults in the United States. Arch Ophthalmol 2004; 122: $477-485$.

2 Sharma S, Oliver-Fernandez A, Liu W, Buchholz P, Walt J. The impact of diabetic retinopathy on health-related quality of life. Curr Opin Ophthalmol 2005; 16: 155-159.

3 Brown MM, Brown GC, Sharma S, Shah G. Utility values and diabetic retinopathy. Am J Ophthalmol 1999; 128: 324-330.
4 Lamoureux EL, Hassell JB, Keeffe JE. The impact of diabetic retinopathy on participation in daily living. Arch Ophthalmol 2004; 122: 84-88.

5 Mackenzie PJ, Chang TS, Scott IU, Linder M, Hay D, Feuer WJ et al. Assessment of vision-related function in patients with age-related macular degeneration. Ophthalmology 2002; 109: 720-729.

6 Cusick M, SanGiovanni JP, Chew EY, Csaky KG, HallShimel K, Reed GF et al. Central visual function and the NEI-VFQ-25 near and distance activities subscale scores in people with type 1 and 2 diabetes. Am J Ophthalmol 2005; 139: 1042-1050.

7 Mangione CM, Lee PP, Gutierrez PR, Spritzer K, Berry S, Hays RD et al. National Eye Institute Visual Function Questionnaire Field Test Investigators. Development of the 25-item national eye institute visual function questionnaire. Arch Ophthalmol 2001; 119: 1050-1058.

8 Mangione CM, Lee PP, Pitts J, Gutierrez P, Berry S, Hays RD. Psychometric properties of the national eye institute visual function questionnaire (NEI-VFQ). NEI-VFQ field test investigators. Arch Ophthalmol 1998; 116: 1496-1504.

9 Mangione CM, Phillips RS, Seddon JM, Lawrence MG, Cook EF, Dailey R et al. Development of the 'activities of daily vision scale'. A measure of visual functional status. Med Care 1992; 30: 1111-1126.

10 Rosen ES, Mamalis N. Visual Acuity Chart. J Cat Ref Surg 2008; 35: A4.

11 Bass EB, Marsh MJ, Mangione CM, Bressler NM, Childs AL, Dong LM et al. Submacular Surgery Trials Research Group. Patients' perceptions of the value of current vision: assessment of preference values among patients with subfoveal choroidal neovascularization - the submacular surgery trials vision preference value scale: SST report no. 6 . Arch Ophthalmol 2004; 122: 1856-1867.

12 Froberg DG, Kane RL. Methodology for measuring healthcare preferences, II: scaling methods. J Clin Epidemiol 1989; 42(5): 459-471.

13 Ryan M, Scott DA, Reeves C, Bate A, van Teijlingen ER, Russell EM et al. Eliciting public preferences for healthcare: a systematic review of techniques. Health Technol Assess 2001; 5(5): 1-186.

14 Margo CE, Mack WP. Therapeutic decisions involving disparate clinical outcomes: patient preference survey for treatment of central retinal artery occlusion. Ophthalmology 1996; 103: 691-696.

15 Jampel HD, Schwartz GF, Robin AL, Abrams DA, Johnson E, Miller RB. Patient preferences for eye drop characteristics: a willingness-to-pay analysis. Arch Ophthalmol 2003; 121: 540-546.

16 Klein R, Moss SE, Klein BE, Gutierrez P, Mangione CM. The NEI-VFQ-25 in people with long-term type 1 diabetes mellitus: the Wisconsin epidemiologic study of diabetic retinopathy. Arch Ophthalmol 2001; 119: 733-740.

17 Brown GC, Brown MM, Sharma S. Difference between ophthalmologists' and patients' perceptions of quality of life associated with age-related macular degeneration. Can J Ophthalmol 2000; 35: 127-133.

18 Brown MM, Brown GC, Sharma S. Value-based medicine and vitreoretinal diseases. Curr Opin Ophthalmol 2004; 15: 167-172.

19 Busbee BG, Brown GC, Brown MM. Value-based medicine: a new paradigm to evaluate treatments for vitreoretinal diseases and other medical interventions. Int Ophthalmol Clin 2004; 44: 155-172. 
20 Ko GJ, Brown MM, Brown GC. The macroeconomics of vitreoretinal diseases. Curr Opin Ophthalmol 2005; 16: 184-190.

21 Ghazi-Nouri SM, Tranos PG, Rubin GS, Adams ZC, Charteris DG. Visual function and quality of life following vitrectomy and epiretinal membrane peel surgery. $\mathrm{Br} \mathrm{J}$ Ophthalmol 2006; 90: 559-562.
22 Childs AL, Submacular Surgery Trials Research Group. Effect of order of administration of health-related quality of life interview instruments on responses. Qual Life Res 2005; 14: 493-500.

23 Coyne KS, Margolis MK, Kennedy-Martin T, Baker TM, Klein R, Paul MD et al. The impact of diabetic retinopathy: perspectives from patient focus groups. Fam Pract 2004; 21: 447-453. 